\title{
ESTIMATIVAS DE DOSE EFETIVA OCUPACIONAL EM UMA INSTALAÇÃO NUCLEAR COM UM REATOR PWR HIPOTÉTICO DE PEQUENO PORTE
}

\author{
João Domingos Talon \\ Instituto Militar de Engenharia (IME) \\ Praça General Tibúrcio, 80, Urca - Rio de Janeiro - RJ \\ lutalon@yahoo.com.br \\ Alberto Teixeira Neto \\ Instituto Militar de Engenharia (IME) \\ Praça General Tibúrcio, 80, Urca - Rio de Janeiro - RJ \\ albertoneto@ime.eb.br \\ Thomaz Jacintho Lopes \\ Instituto Militar de Engenharia (IME) \\ Praça General Tibúrcio, 80, Urca - Rio de Janeiro - RJ \\ thomazjlopes@ime.eb.br \\ Marcos Paulo Cavaliere \\ Instituto Militar de Engenharia (IME) \\ Praça General Tibúrcio, 80, Urca - Rio de Janeiro - RJ \\ eng.cavaliere@gmail.com \\ João Vitor Mendes da Silva \\ Instituto Militar de Engenharia (IME) \\ Praça General Tibúrcio, 80, Urca - Rio de Janeiro - RJ \\ j27_vitor@gmail.com
}

\begin{abstract}
RESUMO
Neste trabalho, um reator nuclear modular de pequeno porte (SMR) ${ }^{1}$, hipotético do tipo $\mathrm{PWR}^{2}$, com enriquecimento do combustível de $20 \%$ e potência de $50 \mathrm{MWe} / 150 \mathrm{MWt}^{3}$, foi simulado no contexto de uma condição de criticalidade, assim como de supercriticalidade provocada devido à ausência de Boro no moderador. A $300 \mathrm{~cm}$ do vaso de pressão do reator foi medida a dose anual de 1,38E+9mSv para a condição de criticalidade, que ocasionou a necessidade de calcular e instalar uma blindagem no contexto do plano de proteção radiológica da instalação nuclear para fins de licenciamento e cumprimento das diretrizes pertinentes, decorrentes de uma análise onde se prevê um acidente nuclear. Os dados disponíveis também foram utilizados para o dimensionamento do projeto básico do serviço de proteção radiológica da instalação. Os resultados podem, ainda, ser utilizados na elaboração do respectivo PSAR ${ }^{4}$.
\end{abstract}

Palavra-chave: Dose efetiva; SMR; SCALE; Radioproteção; Blindagem.

\footnotetext{
${ }^{1}$ SMR: Small Modular Reactor.

${ }^{2}$ PWR: Pressurized Water Reactor.

${ }^{3}$ MWe: megawatt elétrico e MWt: megawatt térmico.

${ }^{4}$ PSAR: Preliminary Safety Analysis Report.
} 


\begin{abstract}
In this work a small modular nuclear reactor (SMR) with a fuel enrichment of $20 \%$ and power of 50MWe/150MWt was simulated in the context of a criticality as well as supercriticality condition caused by the absence of Boron in the moderator. At $300 \mathrm{~cm}$ from the reactor pressure vessel a dose of $1.38 \mathrm{E}+9 \mathrm{mSv}$ was measured for the criticality condition, which led to the need to calculate and install shielding in the context of the nuclear facility's radiological protection plan for licensing and compliance with guidelines from an analysis of a nuclear accident. The available data was also used to the basic project of the facility's radiation protection service. The results can also be used in the elaboration of the respective PSAR.
\end{abstract}

Keywords: Effective dose; SMR; SCALE; Radioprotection; Shielding.

\title{
1. INTRODUÇÃO
}

O estudo apresenta um SMR, hipotético do tipo PWR, com potência de 50MWe/150MWt, que pode ser aplicado para a geração de energia destinada ao uso comercial e também para outras finalidades, tais como dessalinização da água do mar e propulsão de meios navais, devido ao seu baixo custo de investimento inicial, maior flexibilidade de instalação e maior eficiência em relação aos tradicionais reatores nucleares (United States Department of Energy - DOE) [9]. Em função do elevado enriquecimento, na ordem de 20\%, do elemento combustível (EC) se prevê a necessidade de manutenção do reator com frequência bianual, em média, para troca do EC e outras correções que se fizerem necessárias.

Foi considerada a ocorrência de um acidente de supercriticalidade durante o processo de manutenção do reator e comparada à situação de rotina de seu funcionamento. Dessa maneira, é de suma importância verificar a abrangência do impacto dos danos ocasionados aos Indivíduos Ocupacionalmente Expostos (IOE), bem como as instalações nucleares. Assim, o núcleo desse reator foi modelado e simulado utilizando como ferramenta o software SCALE (KENO VI e T6-DEPL) [7] visando calcular a atividade média de forma a definir os requisitos mínimos para radioproteção dos IOE e os aspectos de segurança nas instalações nucleares [6].

O objetivo deste trabalho foi estimar a dose efetiva ocupacional, em pontos de interesse, a fim de verificar a distância na qual a blindagem de radioproteção da instalação nuclear deve ser implementada.

\section{METODOLOGIA}

A metodologia apresenta o estudo fundamentado na descrição da dose efetiva recebida pelo IOE durante a manutenção da instalação nuclear mencionada. Inicialmente, foi definido o núcleo do reator, conforme as Figuras 1 e 2. Utilizou-se o software SCALE/KENO VI com a finalidade de modelar a geometria do núcleo e o T6-DEPL para obter as atividades médias decorrentes dos radionuclídeos provenientes das reações nucleares ocorridas durante a queima do material físsil ao longo de um dia de operação do reator.

Os parâmetros nucleares, considerando a condição de criticalidade de funcionamento do reator no ciclo zero, foram: temperatura do combustível e da água T=613K, 700ppm de Boro diluído no moderador e composição isotrópica do elemento combustível $\mathrm{UO}_{2}$, com enriquecimento a $20 \%$ do ${ }_{92}^{235} \mathrm{U}$. Observa-se, também, a presença de combustível queimável $\mathrm{UO}_{2}+\mathrm{Gd}_{2} \mathrm{O}_{3}$ no núcleo do reator. 


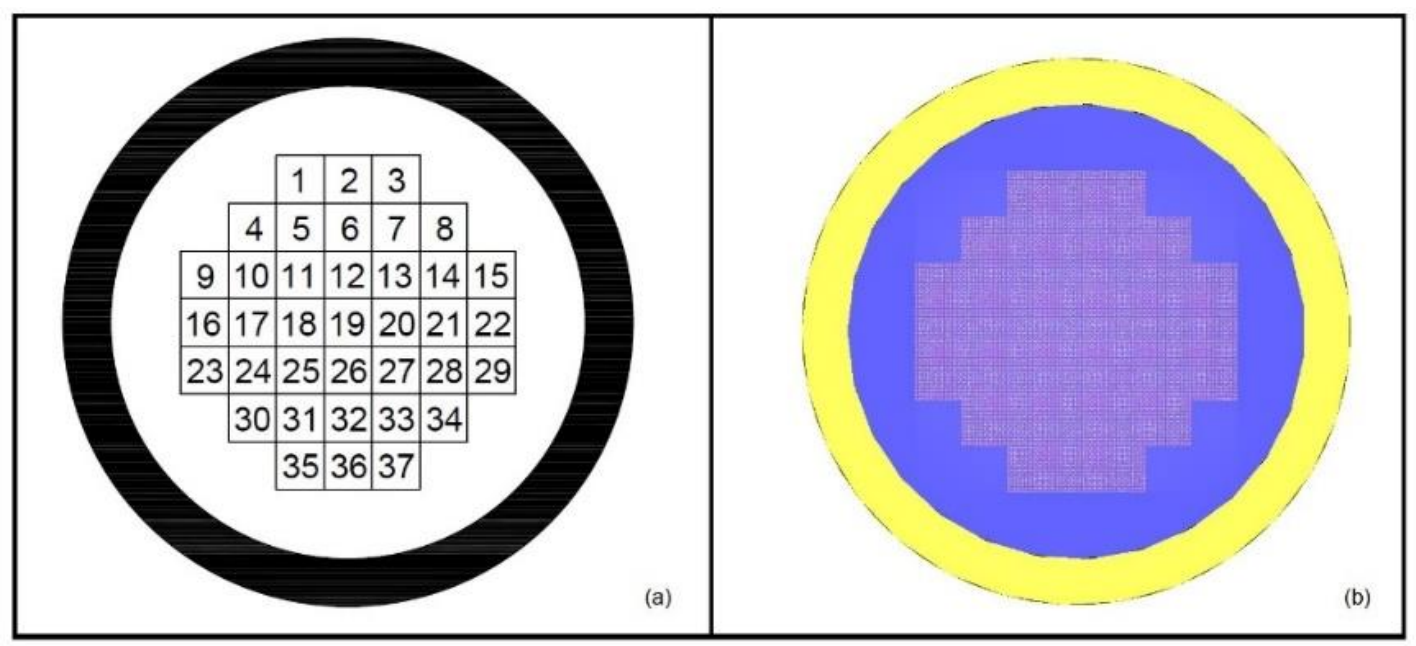

Figura 1 - Vista superior do núcleo de reator (a) AutoCAD, (b) SCALE/KENO VI.

Na condição de supercriticalidade de funcionamento do reator foram observados os mesmos parâmetros nucleares da condição de criticalidade, exceto o Boro, considerado ausente no moderador.

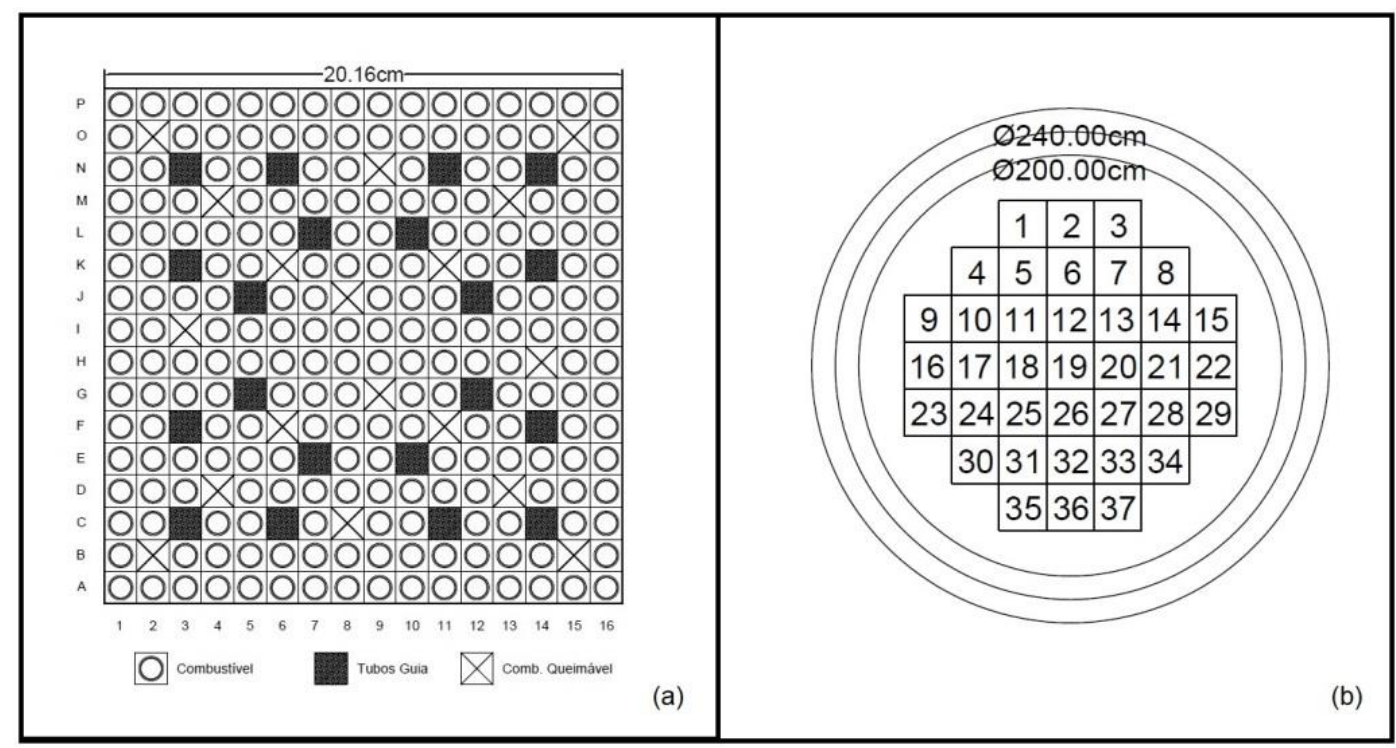

Figura 2 - Vistas superiores: (a) elemento combustível, (b) configuração dos elementos combustíveis no núcleo.

A partir dos resultados das atividades obtidas por meio da simulação do núcleo do SMR utilizando o T6-DEPL, considerou-se uma fonte pontual e isotrópica emitindo fótons monoenergéticos, conforme a Figura 3. 


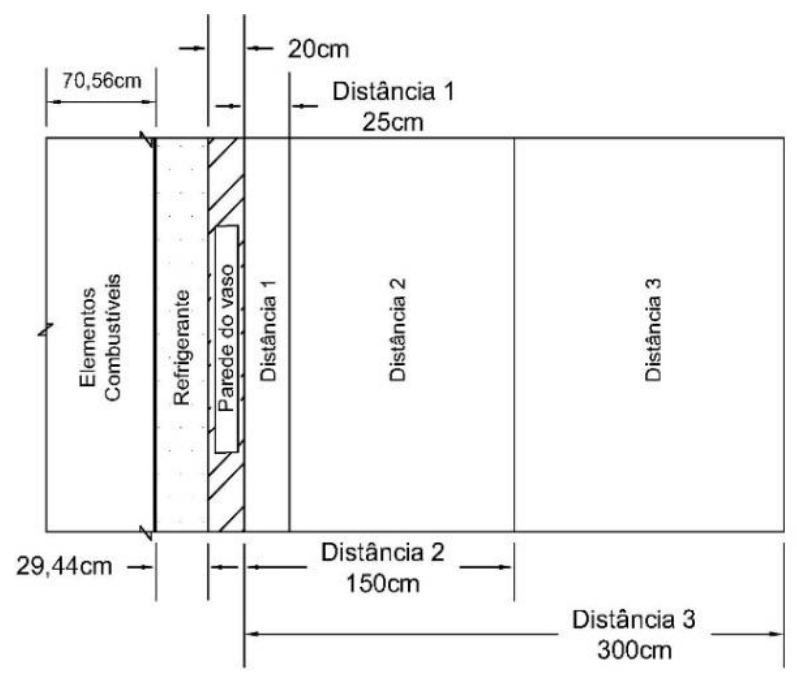

Figura 3 - Camadas percorridas pelos fótons.

A lei da atenuação exponencial, apresentada na Equação (3.1), foi utilizada considerando o arranjo apresentado na Figura 3, que é de má geometria ${ }^{5}$, acarretando a necessidade de considerar o efeito de buildup [8], apresentado no decorrer da trajetória dos fótons.

$$
I=I_{0} \cdot e^{-x} \cdot B(\mu x)
$$

Foram calculados os fatores de buildup de Taylor $B_{T}(\mu x)$ [1][4], para meio infinito, considerando os diferentes materiais, individualmente, a partir da Equação (3.2).

$$
B_{T}(\mu x)=A_{1} \cdot e^{-\mu_{1} \cdot x}+A_{2} \cdot e^{-\mu_{2} \cdot x}
$$

O trajeto da radiação passa por diferentes materiais, conforme a Figura 3: cladding, água, parede do vaso de pressão e ar. Calculados os $B_{T}(\mu x)$, determinou-se de forma linear, para considerar o acréscimo total de radiação devido ao duplo espalhamento Compton ocorrido nas interações da radiação com os supracitados materiais, o fator de buildup de Broder $\left(B_{B}\right)$ [3], mostrado na Equação (3.3).

$$
B_{B}\left(\mu_{1} \cdot x_{1} ; \mu_{2} \cdot x_{2} ; \mu_{3} \cdot x_{3}\right)=\left[B_{1}\left(l_{1}\right)+B_{2}\left(l_{1}+l_{2}\right)+B_{3}\left(l_{1}+l_{2}+l_{3}\right)\right]-\left[B_{2}\left(l_{1}\right)+B_{3}\left(l_{1}+l_{2}\right)\right]
$$

Com o cálculo do fator de transmissão $(F T)$, determinado por meio da relação $I / I_{0}$ e, a partir da atividade total inicial $\left(A_{0}\right)$, obtida por meio da simulação, foi calculada a atividade na parede externa do vaso do reator $(A)$. Deste modo, foi obtida a dose absorvida [1] [3] pela equação a seguir:

$$
D=\frac{A}{4 \pi r^{2}}\left[\sum_{i}^{\infty} E_{i}\left(\frac{\mu}{\rho}\right)_{i}\right]
$$

A dose equivalente [8], em sievert (Sv), é dada por:

$$
H=D \cdot Q
$$

\footnotetext{
${ }^{5}$ Má geometria: consideração realizada devido ao feixe incidente de radiação não ser colimado ao longo de sua trajetória.
} 
onde, Q representa o fator de qualidade considerado como 1, pois foram considerados apenas fótons.

A dose efetiva [8] é obtida por meio de:

$$
E=\sum_{T} W_{T} H_{T}
$$

Como foi considerada a dose absorvida para o corpo inteiro, matematicamente, a dose equivalente se iguala a dose efetiva.

Os resultados obtidos nas simulações foram comparados com os limites estabelecidos pelo órgão regulador - Comissão Nacional de Energia Nuclear (Cnen) - para um IOE quando exposto a um acidente radiológico cujo valor máximo da dose efetiva é de $100 \mathrm{mSv}$.

Os valores calculados para o coeficiente de atenuação $(\mu)$ e coeficiente de atenuação de massa $\left(\frac{\mu_{e n}}{\rho}\right)$ foram extrapolados por meio de funções polinomiais visando maior aderência à tendência dos valores tabelados [4].

Para o cálculo da massa do material a ser empregado na blindagem foi considerado o reator inserido em um envoltório de base e cobertura de $(840 \times 840) \mathrm{cm}$, altura de $750 \mathrm{~cm}$ e a espessura do material a ser utilizado.

As áreas são classificadas em [6]:

1. Área controlada - área sujeita a regras especiais de proteção e segurança, com a finalidade de controlar as exposições normais, prevenir a disseminação de contaminação radioativa e prevenir ou limitar a amplitude das exposições potenciais;

2. Área supervisionada - área para a qual as condições de exposição ocupacional são mantidas sob supervisão, mesmo que medidas de proteção e segurança específicas não sejam normalmente necessárias; supervisionada.

3. Área livre - qualquer área que não seja classificada como área controlada ou área

Ressalta-se que não foram considerados os nêutrons que fogem através da parede externa do reator, assim como os raios- $\mathrm{X}$ característicos emitidos por todos os materiais empregados no reator e na blindagem.

\section{RESULTADOS}

Realizadas as simulações nas condições de criticalidade e supercriticalidade, foram obtidos os radionuclídeos e suas respectivas atividades como resultado da queima do núcleo do reator ao longo de um dia, cujo somatório foi a atividade total $\left(A_{0}\right)$.

Para o reator na condição de criticalidade foram considerados todos os emissores de fótons após a queima. Com isso, obteve-se as respectivas energias por meio do LARAWEB [5], conforme descrito no Quadro 1. 


\begin{tabular}{|c|c|c||c|c|c|}
\hline Radionuclídeo & Atividade $(\mathrm{Bq})$ & Energia $(\mathrm{keV})$ & Radionuclídeo & Atividade $(\mathrm{Bq})$ & Energia $(\mathrm{keV})$ \\
\hline Am241 & $9,45 \mathrm{E}+00$ & 59,541 & Pu241 & $8,82 \mathrm{E}+06$ & 16,167 \\
\hline Am242m & $5,96 \mathrm{E}-04$ & 18,080 & Pu242 & $1,86 \mathrm{E}-02$ & 16,675 \\
\hline Am243 & $2,94 \mathrm{E}-03$ & 74,660 & Pu243 & $1,00 \mathrm{E}+03$ & 84,000 \\
\hline Cm242 & $7,34 \mathrm{E}+01$ & 17,595 & Sm151 & $3,43 \mathrm{E}+10$ & 6,483 \\
\hline Cm243 & $7,34 \mathrm{E}-01$ & 277,599 & Tc99 & $5,77 \mathrm{E}+07$ & 89,520 \\
\hline Cm244 & $1,18 \mathrm{E}+00$ & 17,055 & U234 & $1,17 \mathrm{E}+10$ & 15,311 \\
\hline Cm245 & $9,75 \mathrm{E}-07$ & 103,734 & U235 & $1,50 \mathrm{E}+10$ & 185,720 \\
\hline Pu238 & $9,03 \mathrm{E}+04$ & 16,167 & U236 & $4,11 \mathrm{E}+07$ & 15,359 \\
\hline Pu239 & $4,89 \mathrm{E}+09$ & 16,167 & U238 & $9,35 \mathrm{E}+09$ & 15,311 \\
\hline Pu240 & $2,04 \mathrm{E}+07$ & 16,167 & Atividade Total (A $)(\mathrm{Bq})=7,54 \mathrm{E}+10$ \\
\hline
\end{tabular}

Quadro 1 - Radionuclídeo, atividade e energia para o núcleo do reator na condição de criticalidade.

Por meio da Equação (3.6), obteve-se as doses efetivas em diversos pontos de interesse numa instalação nuclear para corpo inteiro para um homem padrão de $70 \mathrm{~kg}$, cujos resultados estão apresentados no Quadro 2.

\begin{tabular}{|c|c|c|c|c|c|}
\hline Distância $(\mathrm{cm})$ & 25 & 150 & 300 & 400 & 427,8 \\
\hline Taxa de Dose (mSv/s) & 27534,27 & 764,84 & 191,21 & 107,56 & 94,03 \\
\hline Dose (mSv/ano) & $1,98 \mathrm{E}+11$ & $5,51 \mathrm{E}+9$ & $1,38 \mathrm{E}+9$ & $7,74 \mathrm{E}+8$ & $6,77 \mathrm{E}+8$ \\
\hline
\end{tabular}

Quadro 2 - Doses efetivas nos pontos de interesse para o reator na condição de criticalidade.

Considerando que o limite máximo normativo (média de cinco anos) da dose efetiva anual para um IOE é de $20 \mathrm{mSv}$ [6] para a condição de rotina e tomando como referência, neste trabalho, uma distância de $300 \mathrm{~cm}$ da parede externa do vaso do reator para o IOE, segundo os cálculos efetuados, prevê-se uma dose efetiva anual de 1,38E+9mSv. Portanto, é necessário colocar uma blindagem entre a fonte de radiação e o IOE.

Similarmente, para o reator na condição de supercriticalidade os dados obtidos estão representados no Quadro 3.

\begin{tabular}{|c|c|c||c|c|c|}
\hline Radionuclídeo & Atividade $(\mathrm{Bq})$ & Energia $(\mathrm{keV})$ & Radionuclídeo & Atividade $(\mathrm{Bq})$ & Energia $(\mathrm{keV})$ \\
\hline Am241 & $3,62 \mathrm{E}+00$ & 59,541 & Pu241 & $3,37 \mathrm{E}+06$ & 16,167 \\
\hline Am242m & $2,05 \mathrm{E}-04$ & 18,080 & Pu242 & $6,98 \mathrm{E}-03$ & 16,675 \\
\hline Am243 & $3,10 \mathrm{E}-03$ & 74,660 & Pu243 & $277,40 \mathrm{E}+00$ & 84,000 \\
\hline Cm242 & $7,80 \mathrm{E}+01$ & 17,595 & Sm151 & $3,49 \mathrm{E}+10$ & 6,483 \\
\hline Cm243 & $7,81 \mathrm{E}-01$ & 277,599 & Tc99 & $5,92 \mathrm{E}+7$ & 89,520 \\
\hline Cm244 & $1,26 \mathrm{E}+00$ & 17,055 & $\mathrm{U} 234$ & $1,25 \mathrm{E}+10$ & 15,311 \\
\hline Cm245 & $6,88 \mathrm{E}-07$ & 103,734 & $\mathrm{U} 235$ & $1,60 \mathrm{E}+10$ & 185,720 \\
\hline Pu238 & $5,70 \mathrm{E}+04$ & 16,167 & $\mathrm{U} 236$ & $3,43 \mathrm{E}+7$ & 15,359 \\
\hline Pu239 & $3,18 \mathrm{E}+09$ & 16,167 & U238 & $9,95 \mathrm{E}+9$ & 15,311 \\
\hline Pu240 & $9,23 \mathrm{E}+06$ & 16,167 & \multicolumn{2}{|c|}{ Atividade Total $\left(\mathrm{A}_{0}\right)(\mathrm{Bq})=7,66 \mathrm{E}+10$} \\
\hline
\end{tabular}

Quadro 3 - Radionuclídeo, atividade e energia para o núcleo do reator na condição de supercriticalidade. 
Foram calculadas as doses efetivas conforme dados do Quadro 4.

\begin{tabular}{|c|c|c|c|c|c|}
\hline Distância $(\mathrm{cm})$ & 25 & 150 & 300 & 400 & 427,8 \\
\hline Taxa de Dose (mSv/s) & 29301,89 & 813,94 & 203,49 & 114,46 & 100,00 \\
\hline Dose (mSv/ano) & $2,11 \mathrm{E}+11$ & $5,86 \mathrm{E}+9$ & $1,47 \mathrm{E}+9$ & $8,24 \mathrm{E}+8$ & $7,20 \mathrm{E}+8$ \\
\hline
\end{tabular}

Quadro 4 - Doses efetivas nos pontos de interesse para o reator na condição de supercriticalidade.

No caso de um acidente a norma autoriza uma dose efetiva de até 100mSv [6] para IOE voluntário trabalhar nesta condição, que está fora da condição de rotina prevista. Essa condição é devido à ocorrência de uma supercriticalidade, conforme os dados do Quadro 4. Logo, é necessário prever a colocação de blindagem que permita a presença de IOE.

Conforme mencionado, adotou-se a distância de $300 \mathrm{~cm}$ da parede externa do vaso do reator até o IOE. Foi, então, calculada a espessura da blindagem do reator na condição de criticalidade utilizando chumbo ou ferro ou concreto, por serem os materiais comumente utilizados nesse tipo de blindagem, visando atender à norma em vigor para instalações nucleares. Em seguida, calculou-se a massa a fim de verificar quanto de material seria empregado na construção da blindagem. Os valores obtidos estão no Quadro 5.

\begin{tabular}{|c|c|c|c|}
\hline Materiais & Chumbo & Ferro & Concreto \\
\hline Espessura da blindagem (cm) & 3,68 & 22,23 & 86,90 \\
\hline Massa (ton) & 164,05 & 687,01 & 819,89 \\
\hline
\end{tabular}

Quadro 5- Blindagem e massa para a condição de critilicalidade.

Na condição de supercriticalidade, caso ocorra o acidente em estudo e utilizando a blindagem apresentada no Quadro 5, foi calculada a dose efetiva no valor de 20,68mSv, abaixo da dose de $100 \mathrm{mSv}$ para a condição de acidente.

Com os dados obtidos, pode-se classificar as áreas em:

1. Área controlada - área da blindagem até o reator onde o IOE está sujeito a exposição superior a 100mSv. O IOE, então, estaria sujeito a uma dose além da dose máxima prevista para o caso de acidente ou incidente, condição prevista somente para o caso do IOE voluntário;

2. Área supervisionada - área da blindagem até $126 \mathrm{~cm}$, onde o IOE está sujeito a exposição entre $20 \mathrm{mSv}$ e $1 \mathrm{mSv}$;

3. Área livre - área além de $126 \mathrm{~cm}$ da blindagem.

\section{CONCLUSÕES}

Na distância de $300 \mathrm{~cm}$ da parede externa do vaso do reator obteve-se a dose efetiva anual de $1,38 \mathrm{E}+9 \mathrm{mSv}$ e de $1,47 \mathrm{E}+9 \mathrm{mSv}$ para as condições de criticalidade e supercriticalidade, respectivamente. Do exposto, verificou-se a necessidade de aplicação de blindagem em torno da instalação nuclear de modo que a dose, além dessa blindagem, atenda aos limites estabelecidos na norma CNEN 3.0.1 (Diretrizes Básicas de Proteção Radiológica).

Assim, conforme o Quadro 5, para um projeto em que a blindagem foi estabelecida na distância de $300 \mathrm{~cm}$ em relação à parede externa do vaso do reator, obteve-se as espessuras de blindagem de $3,68 \mathrm{~cm}, 22,23 \mathrm{~cm}$ e $86,90 \mathrm{~cm}$ para o chumbo, ferro e concreto, respectivamente. Também, conforme as massas calculadas, verifica-se que a tonelagem de chumbo é, aproximadamente, quatro vezes menor do que a do ferro e cinco vezes menor do que a do concreto. Portanto, caso a instalação nuclear seja aplicada a propulsão de um meio naval, o chumbo deverá ser o material empregado na blindagem, haja vista o menor impacto na tonelagem do projeto. 
A blindagem estabelecida na instalação nuclear utilizando quaisquer dos materiais mencionados, considerando o reator na condição de criticalidade, atende também a situação de acidente, haja vista que a dose efetiva a $300 \mathrm{~cm}$ da parede externa do reator $(20,68 \mathrm{mSv})$ está abaixo de $100 \mathrm{mSv}$, atendendo à norma em vigor para a condição de acidente. Além disso, verifica-se que a área supervisionada está compreendida entre a parede externa da blindagem e a distância de $126 \mathrm{~cm}$ da mesma e a área livre além dessa distância.

Portanto, constatou-se que a metodologia apresentada pode ser aplicada em apoio ao projeto de blindagem que atenda as normas em vigor no que tange à dose efetiva assim como à segurança do IOE de uma instalação nuclear.

\section{REFERÊNCIAS}

[1] Attix, F.H.. Introduction Radiological Physics and Radiation Dosimetry. WileyVCH Verlag GmbH \& Co, 2004.

[2] AUTOCAD.

Disponível

em: <https:www.autodesk.com.br/products/autocad/overview> Acesso em: 15 de maio de 2019.

[3] Chilton, A. B.; Shultis, J. K.; Faw, R. E.. Principles of Radiation Shielding. PrenticeHall, INC. Englewood Cliffs, NJ, EUA, 1984.

[4] Foderaro, A.. The Photon Shielding Manual. University Park Pennsylvania, PA, EUA, 1976.

[5] LARAWEB. Disponível em: < http://www.nucleide.org/Laraweb/index.php > Acesso em: 19 de maio de 2019.

[6] Norma Cnen 3.0.1: Diretrizes Básicas de Proteção Radiológica, Rio de Janeiro, RJ, aprovada pela resolução 164/14, março de 2014.

[7] Rearden, B. T.; Jessee, M. A. PRIMER SCALE. Code Systems ORNL/TM-2005/39. v. 6.2, Oak Ridge, TN, abril de 2016.

[8] Tauhata, L.; Salati, I.; Di Prinzio, R.; Di Prinzio, A. R.. Radioproteção e Dosimetria: Fundamentos. Comissão Nacional de Energia Nuclear, Instituto de Radioproteção e Dosimetria, Rio de Janeiro, RJ, 10a rev-2014.

[9] United States Department of Energy - DOE (2019). Reactor Technology: Benefits of Small Modular Reactors (SMRs). Disponível em: <https://www.energy.gov/ne/benefitssmall-modular-reactors-smrs>. Acesso em: 12 de agosto de 2019. 